\title{
Drama als Methode: Theaterorientierte Unterrichtsformen in Schule und Universität
}

\author{
Eine Veranstaltungsreihe des Fachdidaktikzentrums der \\ Geisteswissenschaftlichen Fakultät Graz. \\ Karl-Franzens-Universität im Wintersemester 2012/13.
}

\section{Konferenzbericht von Micha Fleiner}

Im Wintersemester 2012/13 fand an der Karl-Franzens-Universität Graz eine Veranstaltungsreihe zum Thema „Drama als Methode: Theaterorientierte Unterrichtsformen in Schule und Universität" statt, die im Zeichen des Forschungsschwerpunktes „Lernen - Bildung - Wissen“ stand. Konzipiert und federführend betreut wurde die Veranstaltungsreihe vom Fachdidaktikzentrum der Geisteswissenschaftlichen Fakultät Graz, dessen zentraler Leitgedanke neben einer transdisziplinär ausgerichteten Lehr-Lern-Forschung die Vorstellung einer zukunftsfähigen Vernetzung zwischen schulischen und hochschulischen Akteuren bildet.

Vor dem Hintergrund gegenwärtiger Forschungsergebnisse der Neurobiologie, die auf eine positive Korrelation zwischen synästhetischen Arbeitsformen und einer nachhaltigen Persönlichkeitsbildung hinweisen, sucht die Veranstaltungsreihe, praxisrelevante Umsetzungsmöglichkeiten dieser Erkenntnisse am Beispiel drama- und theaterpädagogischer Lernformen und -techniken aufzuzeigen. Das Veranstaltungsprogramm folgte hierbei einer zweifachen Stoßrichtung: Die zu Beginn des Wintersemesters organisierte Eröffnungsveranstaltung, die sich einem fachübergreifenden Einblick in forschungsbasierte Fragestellungen zu Drama- und Theaterpädagogik widmete, bildete gleichsam die Grundlage zu einer fachpraktischen Vertiefung im weiteren Verlauf des Semesters in Gestalt thematisch gezielt ausgerichteter Theaterworkshops.

Mit Blick auf die aktuelle Veranstaltungsreihe verwies die Leiterin des Fachdidaktikzentrums, Univ.-Prof.in Dr. Sabine Schmölzer-Eibinger, im Rahmen der Eröffnungsveranstaltung am 19. Oktober denn auch auf die in hohem Maße transformative, d. h. neue Denk- und Wahrnehmungsräume generierende Qualität dramapädagogischer Lehr-Lernprozesse. Für einen verstärkten Einsatz theaterästhetischer Ausdrucks- und Gestaltungsfelder im Bereich der Sprachlehr- und -lernforschung sprach sich in seiner Eröffnungsrede auch Univ.Prof. Dr. David Newby aus. Er begrüßte das verstärkte disziplinübergreifende Interesse an performativen Vermittlungsansätzen als wertvollen Beitrag zu einer zeitgemäßen Unterrichtspraxis im schulischen ebenso wie im hochschulischen Bildungswesen.

Ausgehend von einem fachwissenschaftlichen Überblick über die Entwicklung drama- und theaterbezogener Methodenkonzepte von den Anfängen bis zur 
Gegenwart fokussierten Dr. Ingeborg Ledun-Kahlig und Harald Dier in grundlegendem Sinne die Notwendigkeit eines nachhaltigen Brückenbaus zwischen bewährten Methoden des traditionellen Fremdsprachenunterrichts mit inszenatorisch akzentuierten Zieldimensionen. Ein abschließend vorgenommener Ausblick verdeutlichte die inhaltsreiche Bandbreite der Veranstaltungsreihe, die theoriebasierte Zugänge zu Interaktions- und Inszenierungsmethoden mit praxisorientierten Lösungsansätzen in Form themenzentrierter Workshops zu den Bereichen Educational Drama, Playback-, Forum- und Improvisationstheater konzeptionell verknüpfte.

Am Beispiel einer jüngst im Grazer Raum durchgeführten Studie zu schulischen Theaterworkshops in französischer Sprache reflektierten Barbara Horngacher und Ulrich Kaiser-Kaplaner Hintergründe und Möglichkeiten für den Einsatz dramapädagogischer Methoden im Fremdsprachenunterricht. Anhand der Auswertungsergebnisse des empirisch begründeten Datenmaterials konnte aufgezeigt werden, dass die Mehrheit der in die Untersuchung eingebundenen Lernenden einen signifikanten Zuwachs in Kompetenzdimensionen wie Fremdsprache, performativer Ausdrucksgehalt und Persönlichkeitsbildung erreichte. Eine spontane zielsprachliche Aktivierung aller Veranstaltungsteilnehmerinnen und -teilnehmer vermittelte in diesem Zusammenhang ein lebendiges Bild des mehrdimensional angelegten Forschungsdesigns. Erfreulich war zudem, dass die Referierenden eine bewusste Akzentverlagerung zugunsten des - in der dramapädagogischen Forschungslandschaft erfahrungsgemäß stark unterrepräsentierten - Bereichs der Französischdidaktik legten und auf diese Weise den Anstoß zu einer Ausweitung des fremdsprachendidaktischen Diskussionsfeldes gaben.

Auch Maria Fasching thematisierte die Situation des dramaorientierten Fremdsprachenunterrichts an österreichischen Schulen: Hierzu präsentierte sie Ergebnisse aus ihrem gegenwärtigen Forschungsprojekt, in dessen Rahmen sie die Methode Drama in Education im Englischunterricht des Sekundarschulwesens evaluierte. Anhand fragebogenbasierter Erhebungen unternahm sie eine Perspektivenanalyse österreichischer Schüler/-innen und Fremdsprachenlehrkräfte zu inhaltlichen Kategorien wie Sinngehalt, Kontext, Quantität und Relevanzsetzung dramapädagogischer Unterrichtsangebote. Obschon der offizielle Abschluss der Untersuchung noch aussteht, lassen die vorab dargestellten Ergebnisse bereits erahnen, dass der Einsatz von Drama in Education an den untersuchten Lehranstalten in mehrfacher Hinsicht einen Beitrag zur Qualitätssteigerung des Sprachlehr- und -lernprozesses leisten konnte.

Im Anschluss hieran beleuchtete Micha Fleiner zunächst definitorische Überlagerungen des Begriffes Dramapädagogik und plädierte für eine stärkere begriffliche Konturierung. Mit Blick auf die Rolle der Dramapädagogik im Fremdsprachenunterricht widmete er sich sodann der Frage, welche konkreten Kompetenzen - performative, kognitive, ludisch-affektive - Schülerinnen und Schüler im Rahmen eines dramapädagogisch ausgerichteten Fachunterrichts erwerben können. Überlegungen dieser Art, so Fleiner, implizierten immer auch die grundsätzliche Problematik, über welche Fähigkeiten und Fertigkeiten 
eine Fremdsprachenlehrkraft, die dramapädagogische Lehr-Lern-Prozesse im schulischen Unterricht zu integrieren beabsichtigt, letztlich verfügen sollte. Vor dem Hintergrund eines abschließenden Problemaufrisses über die gegenwärtige Situation der Dramapädagogik im deutschsprachigen Raum unterstrich der Referent mit Nachdruck die Notwendigkeit einer systematischen Implementierung performativ-ästhetischer Kompetenzdimensionen in die hochschulische Ausbildungsphase angehender Fremdsprachenlehrkräfte.

Im Zentrum der gemeinsamen Abschlussdiskussion - angeleitet durch Stefan Egger, einem profunden Kenner und aktiven Mitgestalter der drama- und theaterpädagogischen Szene im österreichischen Raum - standen weiterführende Überlegungen zum spannungsreichen Verhältnis von dramapädagogischer Forschung und Praxis sowie zum Stellenwert einer definitorischen Systematisierung. Den Veranstaltungsteilnehmenden - Studierende, Dozierende, Referierende und praktizierende Lehrkräfte - bot sich auf diese Weise eine interessante Plattform zu einem offenen und konstruktiven Austausch rund um den facettenreichen Themenkomplex Drama als Methode.

Den Auftakt in die dramapädagogische Workshop-Reihe bildete am 22. November Karl Eigenbauer, dessen Veranstaltung eine anwendungsnahe Auseinandersetzung mit Techniken und Methoden des Educational Drama im Kontext des Englischunterrichts fokussierte. Im Modus handlungsgenerierender „Als-ob“-Situationen sensibilisierte Eigenbauer die Teilnehmenden für die Grundlagen kreativer Gestaltungsarbeit ebenso wie für die Potenziale körpersprachlicher Ausdrucksmittel in den Bereichen Gestik, Mimik, Stimme, Kinesik und Proxemik. Ein wesentliches Ziel des Workshops, in dessen Mittelpunkt methodische Arbeitsformen wie still images, hot seating, alter ego, character pot, teacher in role und weitere mehr rückten, bestand darin, Fremdsprachenlehrerinnen und -lehrer zu einem bewussten und reflektierten Einsatz theaterästhetischer Unterrichtsaktivitäten in fremdsprachlichen Lehr-Lern-Settings zu ermutigen.

Mit Daniel Feldhendler ist es der Veranstaltungsleitung gelungen, einen namhaften Experten aus dem Ausland zu gewinnen, der die Entwicklung dramaund psychodramapädagogischer Lehr-Lern-Formen im Bereich der Fremdsprachendidaktik im deutschsprachigen Raum seit ihren Anfängen maßgeblich förderte. Praxisnahe Einblicke in seinen über vier Jahrzehnte gewachsenen Wissens- und Erfahrungsschatz vermittelte Feldhendler denn auch seinen Workshop-Besuchern in Graz am 14. Dezember: Am Beispiel des darstellungsbezogenen Ansatzes „Das Leben in Szene setzen“ illustrierte er, welch vielgestaltige Einsatzmöglichkeiten das Zusammenspiel von Psychodrama mit humanistischen und dramapädagogischen Verfahren dem fremdsprachlichen Fachunterricht eröffnet. In teilnehmerzentrierten Handlungskontexten wurden hierbei einzelne Elemente des Playback-Theaters und der SprachbiographieForschung in kreativ-spielerischer Manier erkundet - dies stets aus dem Blickwinkel der aktuellen Fremdsprachenvermittlung, d. h. in engem Bezug zu kommunikationsbezogenen Zielsetzungen des Gemeinsamen europäischen Referenzrahmens für Sprachen. 
Das Feld der Fremdsprachendidaktik verlassend, thematisierten die beiden abschließenden Workshops performativ motivierte Lehr-Lern-Verfahren unter besonderer Betonung eines fachgebietsübergreifenden Zusammenhanges: So stellte eine aktive Annäherung an die Methoden des Forumtheaters - einer ebenso lehr- wie konfliktreichen Form des demokratisierenden Theaters - das inhaltliche Kernanliegen des Workshops am 11. Januar dar. Hierzu entwickelte Dr. Michael Wrentschur mit den Teilnehmenden biographisch begründete Konfliktszenarien, die zentrale Impulse für eine gemeinsame Inszenierungsarbeit lieferten. Eine vertrauensvolle Arbeitsatmosphäre, die den Prinzipien Partizipation, Kommunikation und Toleranz in besonderem Maße Rechnung trug, ermöglichte ein zwangloses Erproben performativer Lösungswege. Ergänzende Theaterübungen und -spiele zu Motivation, Teamgeist und ästhetischer Gestaltungsarbeit, die den Teilnehmenden nicht zuletzt wertvolle Anregungen für die eigene Lehrpraxis boten, flankierten die anwendungsbezogene Grundausrichtung der Veranstaltung.

Einen inhaltlich bewusst entgrenzenden Akzent setzte die am 25. Januar durchgeführte Abschlussveranstaltung in dem Sinne, als sie theaterbasierte Arbeitsformen und Improvisationstechniken auf das breite Feld der Naturwissenschaften zu übertragen suchte. Elisabeth Krön förderte den situationsadäquaten Einsatz szenisch-dramatischer Lernformen im spezifischen Kontext des - traditionell stark kognitiv geprägten - naturwissenschaftlichen Unterrichts. Hierfür präsentierte sie den Besucherinnen und Besuchern eine breite Palette an motivations- und interaktionsfördernden Übungsformaten - z. B. zu den Kompetenzfeldern Improvisations- und Inszenierungsarbeit oder Rollen- und Figurenentwicklung -, die allesamt dazu dienten, naturwissenschaftliche Lehrinhalte nicht allein auf theoretisch-analytischem, sondern in erster Linie auf leiblich und sinnlich spürbarem Wege zu vermitteln.

Resümierend lässt sich festhalten, dass es den Organisatorinnen und Organisatoren der Veranstaltungsreihe „Drama als Methode: Theaterorientierte Unterrichtsformen in Schule und Universität" - ein besonderer Dank für ihr hohes persönliches Engagement gebührt an dieser Stelle Dr. Ingeborg Ledun-Kahlig und Harald Dier - gelungen ist, ein inhaltlich facettenreiches und qualitativ gehaltvolles Veranstaltungsprogramm zu entwickeln. Neben aufschlussreichen Einblicken in diegegenwärtige Situation der Dramapädagogik - namentlich im österreichischen Raum - verdeutlichte die Veranstaltungsreihe zudem, in welchem Maße es einer engen wechselseitigen Verknüpfung zwischen Schule und Universität nachzuspüren lohnt. Diese Zieldimension ist nicht zuletzt insoweit von erheblichem Belang, als der Grad an fach- und länderübergreifender Vernetzungsfähigkeit dramapädagogischer Akteure im schulischen, hochschulischen und außerschulischen Bereich in maßgeblicher Hinsicht über die zukünftige Entwicklungsdynamik einer performativ-ästhetisch ausgerichteten Fremdsprachendidaktik $\mathrm{zu}$ entscheiden vermag. In diesem Horizont stellte die dramapädagogische Veranstaltungsreihe der Karl-FranzensUniversität Graz einen ebenso notwendigen wie verdienstvollen Impuls für eine dialogorientierte Profilbildung dar. 